\title{
Exploring the Impact of COVID-19 on Mental Health
}

\author{
Yizhen Wang ${ }^{1 *}$ \\ ${ }^{I}$ Chongqing Nankai Secondary School, 400030 Chongqing, China \\ *Corresponding author. Email: guanghua.ren@gecacdemy.cn
}

\begin{abstract}
With the intensively spreading COVID-19 epidemic around the world, people have faced problems and dramatic changes in daily life, being unable to work as usual and living normally as routines. The consequences in that situation may reflect on the mental health of people since they bring people chaotic lifestyles and some distortion. To discover the fact whether people's mental health is influenced by epidemics, it is required to review conducted survey or interviews directly toward people. Therefore, it would be necessary to explore significant influences caused by COVID19. This review focuses several important previous studies that concerns about the effect epidemic on mental health, including causal factors, methodologies and efficient strategies adopted by researchers.
\end{abstract}

Keywords: mental health, mental heal issues, impact, COVID-19, adopted strategies.

\section{INTRODUCTION}

Contemporarily, there already have been research and surveys that concentrate on mental problems caused by epidemics. Such surveys usually contain sufficient statistical information. It is of great significance to clearly recognize the current situation of mental health problems brought out by COVID-19, aiming to help release pressure in a statistical and self-awareness way. Additionally, with sufficient information of this epidemic, the overall development can be delineated comprehensively and clearly. As a common fact we know that diverse ways are taken into consideration when we evaluate the reasons for occurrences of different types of mental problems. In general, these previously explored methods and reasons can be applied to most cases. However, specified explanations about causes of mental issues should be analyzed again for the COVID-19, due to its particular features that vary from usual situations.

One study by Md Mahbub Hossain et al. suggests a few factors associated with mental health problems [1]. The first factor, age, according to Mahbub Hossain, is risky among the younger generation. Younger persons, for example, showed a considerably higher frequency of generalized anxiety and depressive symptoms than older people, according to Huang and colleagues [2]. Furthermore, Wang and colleagues found that the anxiety risk of people over 40 years old was 0.40 times (95 percent CI: 0.16-0.99) that of people under 40 years old, implying that younger people have a higher anxiety risk.
With uncertainty, this review suggests that the rate of anxiety varies between young people and older adults. [3]

Besides, with similar discussion made by researchers in this study, marital status, education, occupation and income, place of living and comorbid physical health problems and etc. are claimed. For example, Liang and colleagues discovered that having less education was associated with poor mental health among young participants [4]. Low education was also linked to poor mental health outcomes, according to Lei and colleagues. Students in senior high school and those with better marks, on the other hand, showed a higher frequency of depression and anxious symptoms, according to Zhou and colleagues [5]. Similarly, Wang and colleagues found that individuals with a bachelor's degree had 0.39 times (95 percent CI: 0.17-0.87) higher risk of depression than those with a master's degree or higher. All these probable causes provide us with a better understanding of mental problems in the epidemic. And the prevalence of COVID-19 is implied owing to the broad survey conducted.

For care workers, those factors associated with mental problems are also evinced in different ways. Jianbo Lai, MSc et al. conducted another study which found the mental problems prevails especially among female care workers in Wuhan, China [6]. The indication is clear that for care workers during the epidemic, the location and gender indeed matter to the degree of stress, depression and insomnia. Specifically, many participants in this study reported anxiety, sadness, and sleeplessness 
symptoms, with more than $70 \%$ reporting psychological distress. During the acute SARS pandemic, 89 percent of health-care professionals in high-risk scenarios showed psychological symptoms, according to recent research. The psychological reaction of health-care personnel to an infectious disease pandemic is complex. Feelings of vulnerability or lack of control, as well as worry about one's own health, the spread of a virus, the health of one's family and others, changes in job, and isolation, can all be sources of distress. COVID-19 is human-to-human transmissible and is linked to a high rate of morbidity. The feeling of personal risk may be heightened by the fact that it is potentially lethal. Additionally, the stress and anxieties of health care personnel are exacerbated by foreseeable supply constraints and a rising inflow of suspected and confirmed cases of COVID-19. Meanwhile, the significance of studying factors lead to mental issues is emphasized. Concentrating on a certain group of people' health condition can be the optimal choice, since it reduces futile and excess caring for people in less severe situations and enhances the efficiency to maximize public health.

In another survey in 2020, Mengting Wu et al. explores more factors associated with parents and educational level of students. The results show that college students' parents are experiencing less anxiety than those with students still in middle school or primary school [7]. What's more, the association between several variables and parental mental health was investigated in this study. In this survey, while previous research has indicated that women are more prone to stress than males and have larger emotional reactions, the study showed no significant gender differences in parental anxiety, sadness, or stress. The explanation for this might be that the results of this study were skewed due to the minimal number of men in the sample. During the COVID-19 epidemic, residents in central China had the most psychological suffering, which was much greater than in other regions, according to prior research. As a result, the mental health of parents in central and non-central China was compared in this study. Parents who were quarantined or whose family members were quarantined for two weeks had higher levels of depression than those who did not have family members quarantined, according to a recent study. Although isolation reduced virus spread, it resulted in negative emotional experiences such as depression and anxiety, according to the study. Parents' mental illnesses can have a substantial impact on their despair, anxiety, and stress levels. On the one hand, the base of mental illness makes parents more sensitive to the surroundings. On the other hand, during the pandemic, parents are unable to visit the doctor as regularly as they would otherwise, further affecting their mood. These hold a common understanding of mental problems in COVID-19 by revealing relations among different variables.

\section{THE EVALUATION METHODOLOGY ON MENTAL ISSUES}

An important element in evaluating mental issues of prevailing diseases, like influenza and coronavirus, is the adopted methodology in analytical research. It is the same for COVID-19 that numerous studies have used similar or closely related means for evaluation. The following part would review several prominent and common types of methods used by different researchers, and statistic models collecting and analyzing those data.

\subsection{Design and Search}

Inclusion criteria is of great significance to this part of research. In Finiki Nearchou et al.'s review published on 16 November 2020, an integrated mixed methods approach was applied, in order to expand the latitude of the field [8]. By using eight databases and searching them in a specific range and requirements, it limits but concentrates the subject of the study. For instance, they require those studies with a population of 18-year-old people. From a review article by Kim Usher et al., moreover, population of 18 years of age are also specially adopted. The same criteria offer the enduring and sustainable research in the future, keeping the consistency among all reviews [9]. Databases in that research shows the similar patterns which most researcher intend to apply. As we notice, mental issues are something tends to be uncertain and hardly predictable because of many external confounding elements. In most studies of COVID-19, the highlighted part in designing and searching is obviously an important one.

\subsection{Measurement}

According to Table 2 in one study [9], questionnaire is the most widely used method in searching mental health problems of public. Among twelve reviewed studies in the table, the number of psychometric tools is eight, occupying the highest frequency of them. Questionnaire are used as well, developed by the researchers. Studies often use multiple methods to hold a comprehensive search and to determine types of mental problems in a precise way, including personal information, depression, anxiety, uncontrollable stress, positive support from others, etc. Some other narrative reviews use PICO strategy to research scientifically. According to [10], this literature search was carried out in July 2020 using the databases Google Scholar, PubMed, and Scopus. Only papers written in English and research conducted on people were evaluated as inclusion criteria. Articles in scientific journals, letters to editors, comments, and book chapters were all included as publication types. Their literature search was limited to publications published in the previous year (December 2019-July 2020), whereas the historical backdrop was 
provided without regard to time constraints. All these limitations suggest a sufficiently logic and reasonable analysis for the epidemic.

\subsection{Data synthesis}

Among all the studies, formal narrative approach is commonly used. According to [8], since studies had such a wide range of methods for measuring and reporting mental health outcomes, it was decided that a narrative method to synthesizing findings was preferable to a metaanalytical technique. Specifically, the study adopts relevant tables, diagrams, and figures, including a PRISMA-P flow diagram and a conceptual framework describing the relationship between psychological outcomes and pandemic behaviors, as well as summary tables and presenting the key characteristics of the included articles. Mere quantitative summaries are rarely performed in studies of mental health problems of COVID-19.

From all surveys, other methods of data analysis are also conducted. For example, to maintain methodological integrity, the study [11] uses the COREQ unified criteria for reporting qualitative research were used in the conception and implementation of this study. The openended replies were analyzed qualitatively using a datadriven inductive technique to code content into themes. Thematic analysis establishes a coding system in which codes are organized into recurrent themes that are pertinent to the research issue, providing a framework for organizing qualitative data. This kind of data structuring aids in the creation of meaning from complicated raw data.

\section{STRATEGIES TO FIGHT AGAINST MENTAL HEALTH PROBLEMS}

As COVID-19 prevails all over the world ceaselessly, individuals, communities, organizations and governments have set a large number of solutions and strategies to fight against mental issues. These attempts have been carefully analyzed by different researchers and scientists, in order to offer people more available ways to enhance mental conditions. The following part will review some of the strategies studied or used during the epidemic.

\subsection{Exercise}

According to Shaojuan Hu et al.'s narrative review [12], exercise can be considered as an effective method for individual with mental disorders. By searching within PubMed and Web of Science databases, a literature review has been conducted by them. In addition, the mechanisms of exercise on both anxiety and depression have been demonstrated by that review. Then, the exact suggestions are indicated based the positive role of exercising on various chronic illnesses. Despite studies that concentrates on the how exercise benefits people during COVID-19, other research discovers the current condition of exercise. A study [13] points out that exercise is the most common activity used by 2075 respondents in UK to help with mental well-being, which proves the advantage and prevalence of exercise. In another analysis, [14], exercise's role in older people's mental health is significant. It indicates exercise and physical activity are effective treatments for most chronic conditions, with direct impacts on both mental and physical health. In fact, based on epidemiological evidence of its therapeutic advantages and consideration of the primary molecular mediators involved, exercise has been dubbed the "true polypill." The senior population group deserves special attention, because PA and exercise have significant impacts on the hallmarks of aging and related disorders in older individuals, in addition to the advantages stated above. In this way, exercise benefits and avoids frailty, sarcopenia, falls risk, self-esteem, and depression in older adults.

However, exercises and physical activities are also suggested to be only suitable in a certain amount of time. This is explained by international guidelines of physical practice, that those who engaged in excessive physical activity during the quarantine period, exceeding 2.5 hours per day, had lower life satisfaction. It seems that the physical activity may not be manageable for all people to participate in, since ages, mental conditions and occupations of different people are not able to be predicted.

\subsection{Childcare}

Children are also one significant group of people who urgently require mental support. During the epidemic, elementary education and childcare for younger generation plays a crucial role, owing to the benefits that it allows parents to focus on their jobs with less interruptions, housework and multitasking needs [15]. On the other hand, due to the extreme situation brought by COVID-19, women's working burden increases which would inevitably result in the declination in childcare. According to [16], the closure of schools has imposed a massive burden on parents, and especially on working parents. However, not all parents look after their children in the same way. While other studies have mentioned that men who telecommute are more likely to deal with childcare, and more educated people are more likely to telecommute, the unique data set allowed us to disentangle the effect of working from home from that of parents' education on childcare.

Hence, people face an urgent challenge to pay more attention to childcare. The mental condition constructed previously in the childhood contributes largely to the later development of those kids. With the situation when coronavirus prevails around the world, more methods to 
keep children in a mentally healthy environment are required.

\subsection{Meditation}

With an increasing problem of anxiety and depression among people, meditation is deemed as effective ways to improve their mental situation. For example, a mindfulness app has been assessed for its effectiveness in relaxing and enhance well-being [17]. Some other studies also reveal the benefits of meditation as a relatively obtainable way for people [18]. From this analysis of meditation, an available solution based on it might be possible. In a study that measures the effectiveness of meditation, researchers point out that trained teachers showed significant improvements in mindfulness skills, affective empathy, harm avoidance, character traits and interoceptive awareness, as well as psychological wellbeing (on scales of autonomy, environmental mastery, and positive relationships with others), anxiety, depression (especially in the low-resilience group), and emotional exhaustion. This directly shows how useful meditation training can be especially during the COVID19 , reducing the pressure level of people.

\subsection{Public Awareness}

One significant strategy for the overall control of people's mental health conditions includes the correct information communication. With the efforts of government and various media, the public's fear and stress are to some extent limited. Suggestions such as declaring what is known and unknown openly and honestly and stick to the facts as much as possible; conveying the consistent and specific information to the public; acknowledging the emotions are listed in the study [19].

The same challenges are also revealed on social medias. In [20], their findings add to the growing body of evidence suggesting inattention plays a key role in the spread of disinformation online and imply that partisanship is not the primary factor preventing individuals from evaluating truth on social media by highlighting the effect of inattention in the context of COVID-19 disinformation (rather than politics). Rather, it appears that the inclination to be sidetracked from accuracy on social media is more widespread. As a result, it appears that more basic characteristics of the social media setting are diverting people's attention away from accuracy.

\subsection{Online treatment}

A prominent feature in China's response to the COVID-19 is its fast connection to the Internet. The accessibility of online treatment is therefore reasonable and considered to be quite convenient. One way to achieve the goal is by using mobile applications. For instance, Ali-pay, a mobile application built by Jack Ma's team and extensively utilized in China, is available to patients. They can next search the internet hospital platform for an online or internet phone consultation. Patients can submit their medical records, which are only visible to the consulting physician. Patients with chronic conditions benefit greatly from having access to competent guidance and a free evaluation. There are also online lectures and materials on health education available to help chronic mental patients avoid recurrence. Citizens can also utilize the information from these sites to learn how to relax at home.

\section{CONCLUSION}

In all above reviews, we can have a thorough understanding of COVID-19's impact on people's mental health problems; strategies that have already been adopted; challenges waiting to conquer; the availability of people's analytical surveys. From all these, the mental problems of people are clearly illustrated, and the influence is considerable to the world.

\section{REFERENCES}

[1] Hossain MM, Tasnim S, Sultana A, et al. Epidemiology of mental health problems in COVID-19: a review. F1000Res. 2020; 9:636. Published $2020 \quad$ Jun 23. doi:10.12688/f1000research.24457.1

[2] Huang Y, Zhao N: Generalized anxiety disorder, depressive symptoms and sleep quality during COVID-19 outbreak in China: a web-based crosssectional survey. Psychiatry Res. 2020; 288: 112954.

[3] Wang Y, Di Y, Ye J, et al.: Study on the public psychological states and its related factors during the outbreak of coronavirus disease 2019 (COVID-19) in some regions of China. Psychol Health Med. 2020:1-10.

[4] Liang L, Ren H, Cao R, et al.: The Effect of COVID19 on Youth Mental Health. Psychiatr Q. 2020; 112.

[5] Zhou SJ, Zhang LG, Wang LL, et al.: Prevalence and socio-demographic correlates of psychological health problems in Chinese adolescents during the outbreak of COVID-19. Eur Child Adolesc Psychiatry. 2020; 1-10.

[6] Lai J, Ma S, Wang Y, et al. Factors Associated with Mental Health Outcomes Among Health Care Workers Exposed to Coronavirus Disease 2019. JAMA Netw Open. 2020;3(3): 203976. doi:10.1001/jamanetworkopen.2020.3976

[7] Wu M, Xu W, Yao Y, et al. Mental health status of students' parents during COVID-19 pandemic and 
its influence factors. Gen Psychiatr. 2020;33(4): e100250. Published 2020 Jul 21. doi:10.1136/gpsych-2020-100250

[8] Nearchou F, Flinn C, Niland R, Subramaniam SS, Hennessy E. Exploring the Impact of COVID-19 on Mental Health Outcomes in Children and Adolescents: A Systematic Review. International Journal of Environmental Research and Public Health. 2020; 17(22):8479. https://doi.org/10.3390/ijerph17228479

[9] Usher, K., Jackson, D., Durkin, J., Gyamfi, N. and Bhullar, N. (2020), Pandemic-related behaviours and psychological outcomes; A rapid literature review to explain COVID-19 behaviours. Int. J. Mental Health Nurs., 29: 1018-1034. https://doi.org/10.1111/inm.12790

[10] Giorgi G, Lecca LI, Alessio F, Finstad GL, Bondanini G, Lulli LG, Arcangeli G, Mucci N. COVID-19-Related Mental Health Effects in the Workplace: A Narrative Review. International Journal of Environmental Research and Public Health. 2020; 17(21):7857. https://doi.org/10.3390/ijerph17217857

[11] Arnetz JE, Goetz CM, Arnetz BB, Arble E. Nurse Reports of Stressful Situations during the COVID19 Pandemic: Qualitative Analysis of Survey Responses. International Journal of Environmental Research and Public Health. 2020; 17(21):8126. https://doi.org/10.3390/ijerph17218126

[12] Hu S, Tucker L, Wu C and Yang L (2020) Beneficial Effects of Exercise on Depression and Anxiety During the Covid-19 Pandemic: A Narrative Review. Front. Psychiatry 11:587557. doi: 10.3389/fpsyt.2020.587557

[13] Coyle, C., Ghazi, H. \& Georgiou, I. The mental health and well-being benefits of exercise during the COVID-19 pandemic: a cross-sectional study of medical students and newly qualified doctors in the UK. Ir J Med Sci 190, 925-926 (2021). https://doi.org/10.1007/s11845-020-02423-z

[14] Jiménez-Pavón, D., Carbonell-Baeza, A., \& Lavie, C. J. (2020). Physical exercise as therapy to fight against the mental and physical consequences of COVID-19 quarantine: Special focus in older people. Progress in cardiovascular diseases, 63(3), 386388. https://doi.org/10.1016/j.pcad.2020.03.009

[15] Craig, L, Churchill, B. Dual-earner parent couples' work and care during COVID-19. Gender Work Organ. 2021; 28(S1): 514- 527. https://doi.org/10.1111/gwao.12497

[16] Del Boca, D., Oggero, N., Profeta, P. et al. Women's and men's work, housework and childcare, before and during COVID-19. Rev Econ Household 18, 1001-1017 (2020). https://doi.org/10.1007/s11150020-09502-1

[17] O’Donnell, K.T., Dunbar, M. \& Speelman, D.L. Effectiveness of using a meditation app in reducing anxiety and improving well-being during the COVID-19 pandemic: A structured summary of a study protocol for a randomized controlled trial. Trials 21, $1006 \quad$ (2020). https://doi.org/10.1186/s13063-020-04935-6

[18] Behan, C. (2020). The benefits of meditation and mindfulness practices during times of crisis such as COVID-19. Irish Journal of Psychological Medicine, 37(4), 256-258. doi:10.1017/ipm.2020.38

[19] Finset A, Bosworth H, Butow P, et al. Effective health communication - a key factor in fighting the COVID-19 pandemic. Patient Educ Couns. 2020;103(5):873-876. doi: 10.1016/j.pec.2020.03.027

[20] Gao J, Zheng P, Jia Y, Chen H, Mao Y, et al. (2020) Mental health problems and social media exposure during COVID-19 outbreak. PLOS ONE 15(4): e0231924. https://doi.org/10.1371/journal.pone.0231924 